\title{
TTR
}

Traduction, terminologie, re?daction

\section{Étude de terminologie juridique comparée : les notions de gouvernement et d'administration en anglais et en français}

Raymond Pepermans

Volume 5, numéro 1, 1er semestre 1992

La pédagogie de la traduction : questions actuelles (1) et Miscellanées traductologiques

URI : https://id.erudit.org/iderudit/037112ar

DOI : https://doi.org/10.7202/037112ar

Aller au sommaire du numéro

Éditeur(s)

Association canadienne de traductologie

ISSN

0835-8443 (imprimé)

1708-2188 (numérique)

Découvrir la revue

Citer cet article

Pepermans, R. (1992). Étude de terminologie juridique comparée : les notions de gouvernement et d'administration en anglais et en français. TTR, 5(1),

183-193. https://doi.org/10.7202/037112ar d'utilisation que vous pouvez consulter en ligne. 


\section{Étude de terminologie juridique comparée: les notions de gouvernement et d'administration en anglais et en français}

\section{Raymond Pepermans}

Les traducteurs sont conscients de la difficulté de rendre correctement certains termes appartenant au vocabulaire le plus courant, particulièrement lorsqu'ils ont affaire à deux langues apparentées sur le plan linguistique comme l'anglais et le français. Ces langues présentent, en effet, un certain nombre de termes similaires, acquis par emprunt réciproque au cours d'une période donnée, ce qui semble à première vue en faciliter la traduction sans avoir recours au dictionnaire. Ainsi, il paraît logique de traduire les termes anglais «government» et «administration» par leurs homologues français «gouvernement» et "administration». Nous verrons, dans cet article, que cette solution n'est pas aussi simple en raison de la polysémie de ces termes dans chacune des deux langues ainsi que de l'absence de recoupements parfaits entre les notions qu'ils véhiculent d'une langue à l'autre.

On constate qu'en anglais comme en français le vocabulaire juridique et constitutionnel établit une distinction entre la fonction gouvernementale et la fonction administrative mais aussi que, dans certains textes officiels, le terme anglais «government» est souvent rendu en français par "administration» plutôt que par "gouvernement». Ainsi, on lit dans la Loi concemant le statut et l'usage des langues officielles au Canada, sanctionnée le 28 juillet 1988, le paragraphe suivant: 
«federal institution includes any of the following institutions of the Parliament or Government of Canada: (a) the Senate, (b) the House of Commons, (c) the Library of Parliament, (d) any federal court, (e) any board, commission or council, or other body or office, established to perform a government function by or pursuant to an Act of Parliament or by or under the authority of the Governor in Council,...»

«institutions fédérales. Les institutions du Parlement et du Gouvernement du Canada, dont le Sénat, la Chambre des communes et la bibliothèque du Parlement, les tribunaux fédéraux, tout organisme, bureau, commission, conseil, office ou autre, chargé de fonctions administratives ou en vertu des attributions du gouvernement en conseil.»1

On peut se demander dans quelle mesure les notions représentées par ces deux séries de termes - «gouvernement", «administration» et «government», «administration» - correspondent d'une langue à l'autre ou s'il existe une différence d'extension sémantique entre les champs notionnels qui se rattachent à ces termes dans le domaine français par rapport au domaine anglais. Pour répondre à cette question, étudions successivement la signification de ces termes dans leur cadre linguistique propre de manière à mettre en évidence les recoupements ou les variations de sens qui les caractérisent.

\section{La polysémie du terme «gouvernement» en français}

En français, le terme «gouvernement» recouvre plusieurs notions. Tantôt il caractérise le régime d'un pays donné, qu'il s'agisse des détenteurs du pouvoir ou du fonctionnement des institutions, tantôt on en fait un synonyme de «pouvoir exécutif». ${ }^{2}$ Il désigne aussi l'ensemble des

1. Canada - An Act respecting the status and use of the official languages of Canada/Loi concernant le statut et l'usage des langues officielles au Canada - Statutes of Canada, 1988/Lois du Canada de 1988. Chap. 38, art. 3 (1).

2. Edmond Jouve, «Gouvernement», Encyclopaedia Universalis, corpus 8 (Paris, Éditions Encyclopeadia Universalis, 1984), p. 720. 
organes directeurs de l'État; il englobe alors le chef de l'État, le responsable de l'exécutif et les ministres. Parfois le chef de l'État en est exclu. $^{3}$

«On appelle ainsi la fonction exercée par ceux des dirigeants d'un État qui sont investis de l'autorité de déterminer l'orientation politique générale du pays, d'assurer ses relations diplomatiques, d'élaborer les règlements nécessaires à l'application des lois et d'assurer - grâce à la disposition d'une force matérielle de contrainte et de services administratifs spécialisés - l'exécution de ces lois.»"

Selon les auteurs, le terme «gouvernement» désigne donc l'ensemble des organes composés par les gouvernements d'un pays, c'est-à-dire le régime monarchique, républicain ou présidentiel, ou bien l'organe ou l'ensemble d'organes qui est investi de la fonction gouvernementale, et aussi, dans un emploi plus restreint, le ministère. Le terme est polysémique en ce sens que nous pouvons distinguer plusieurs notions différentes selon l'extension qu'on lui donne.

\section{La polysémie du terme «administration» en français}

Le terme «administration» désigne un type de fonction, d'activité qui consiste à assurer l'application des lois et la bonne marche des services publics conformément aux directives gouvernementales et, par extension de sens, l'organe qui l'exerce ou le régime qui le gouverne. ${ }^{6}$ En tant

3. Ibid., p. 721.

4. Roland Mane, «la Fonction gouvernementale», Charles Genoux, et al., L'État et les institutions (Paris, Librairie Larousse, Encyclopoche Larousse, 1976), p. 65.

5. Ibid., p. 67.

6. Paul Robert, Le Grand Robert de la langue française, Dictionnaire alphabétique et analogique de la langue française, $2^{\circ}$ édition, vol. 4 (Paris, Dictionnaires Le Robert, 1985), p. 990. V. aussi: Gérard Cornu, dir., Vocabulaire juridique (Paris, Presses Universitaires de 
qu'organe, il évoque l'ensemble des services et des agents groupés sous l'autorité des ministères ou d'une même direction pour exécuter une tâche administrative, et en tant que régime, il désigne plusieurs formes d'administra-tions, notamment l'administration centrale, centralisée et consultative. ${ }^{7}$ Le terme «administration» apparaît alors en syntagme sous la forme d'un composant déterminé par un déterminant adjectival.

La fonction administrative consiste à assurer un service public par le truchement des personnes morales de droit public. Ces personnes morales constituent l'administration.

Le terme «administration» revêt donc un caractère polysémique similaire à celui que nous venons d'analyser dans le cas du terme «gouvernement».

Les rapports entre les notions de «gouvernement» et d'kadministration» en français

Il importe tout d'abord d'établir une distinction entre la fonction gouvernementale et la fonction administrative dans la tradition française.

En tant que synonyme de "pouvoir exécutif», la fonction gouvernementale exerce des compétences administratives puisque les services publics font partie de ce pouvoir, mais il ne faut pas perdre de vue que cette fonction est aussi étroitement liée à la fonction législative puisque les responsables de l'exécutif assurent l'orientation des institutions correspondant à cette fonction dans le cadre du régime parlementaire. ${ }^{8} \mathrm{La}$ fonction gouvernementale n'est donc pas réductible au législatif ni à l'exécutif que le gouvemement cumule d'ailleurs dans le cas des régimes autoritaires.

France, 1987), p. 377; Edmond Jouve, op. cit., p. 720.

7. Paul Robert, op. cit., vol. 1, p. 120. V. aussi: Gérard Comu, op. cit., p. 27.

8. Edmond Jouve, op. cit., p. 722. 
La notion de gouvemement constitue donc une réalité autonome, qui combine certains aspects de l'exécutif et du législatif, et qui doit être envisagée séparément vis-à-vis de la théorie des trois pouvoirs établissant une séparation entre le législatif, l'exécutif et le judiciaire. D'autre part, en français, on ne considère pas le pouvoir judiciaire comme une fonction gouvernementale malgré le fait que, dans la tradition française, cette fonction y participe d'une certaine manière dans le cadre des tribunaux administratifs qui dépendent de la puissance exécutive.

Lorsqu'il est appliqué au pouvoir exécutif, le terme «gouvernement» désigne la fonction la plus élevée de ce pouvoir, visant la direction suprême des affaires publiques, et déterminant l'orientation générale de la politique d'un pays. ${ }^{9} \mathrm{La}$ fonction gouvernementale est donc une fonction de politique générale et la fonction administrative une fonction d'exécution de cette politique par l'intermédiaire d'un ensemble d'organismes créés à cet effet.

Ces deux notions étant précisées, pour le domaine français, voyons quels sont, dans cette langue, les recoupements notionnels qui sont à la source de difficultés pour le traducteur.

Tout d'abord, l'article «gouvernemental» du Vocabulaire juridique de Gérard Cornu ${ }^{10}$ affirme que dans la terminologie de certains auteurs, la fonction gouvernementale est interprétée comme l'ensemble des compétences exercées par le pouvoir exécutif. Ce dernier ne se limite pas à la mise à effet de ces lois, mais consiste à assumer toute la direction des affaires publiques, des services et des polices. Autrement dit, pour ces auteurs, comme nous l'avons dit plus haut à propos du pouvoir exécutif, la fonction gouvernementale inclut la fonction administrative. Cette extension de sens est limitée à quelques auteurs mais elle introduit tout de même une difficulté supplémentaire quant à la distinction entre ces notions.

9. Paul Robert, op. cit., vol. 4, p. 990.

10. Vocabulaire juridique, p. 377. 
De plus, les dictionnaires signalent le fait que le terme «gouvernement» est souvent pris, dans le vocabulaire courant, dans le sens de «administration centrale de l'État». Par exemple, on dira: un employé du gouvernement plutôt que de l'administration. Cette acception, selon le dictionnaire Robert, est néanmoins abusive dans le vocabulaire juridique. ${ }^{11}$ Signalons également, en français, un emploi particulier du terme «administration» dans le sens de gouvernement pour désigner le gouvernement des États-Unis. Par exemple, on parlera de l'Administration Bush. Cette acception du terme provient de l'anglais où le terme "administration» désigne les membres du gouvernement fédéral.

En français, nous avons donc affaire à deux termes polysémiques dont certaines des notions se recoupent partiellement. De plus, une des acceptions du terme «gouvernement», en langage courant, introduit une certaine confusion dans l'esprit de l'utilisateur en ce qui concerne la compréhension de ces notions.

La situation en anglais est encore plus complexe, non seulement à cause de la polyvalence notionnelle du terme "government», mais surtout de celle du vocable «administration». Malgré le fait que les ouvrages spécialisés établissent une distinction entre les notions de fonction gouvernementale et de fonction administrative, semblable à celle qui existe en français, les dictionnaires de langue font état d'une grande complexité dans la désignation de ces notions, "governement» pouvant dans certains cas être pris pour «administration» et vice-versa.

\section{La polysémie du terme kgovernment» en anglais}

Comme son équivalent français, «government», au sens fonctionnel, désigne la fonction d'élaboration de programmes par opposition à l'administration des décisions qui en découlent et, au sens organique,

11. Paul Robert, op. cit., vol. 4, p. 990. 
l'ensemble des organes par lesquels une entité politique exerce son autorité. ${ }^{12}$

Il y a cependant, à propos de ce deuxième sens, une différence notable avec la conception française selon le type d'organes auxquels la définition se rapporte. Lorsque nous avons dit que le terme «gouvernement» désigne l'ensemble des organes directeurs de l'État, il s'agit uniquement, au sens juridique du terme, de l'ensemble des organes du pouvoir exécutif.

La conception américaine, quant à elle, est plus large, car elle inclut dans sa définition l'ensemble des organes et des fonctions représentatives des trois pouvoirs, ce qu'on appelle au Canada et aux États-Unis «the three branches of government». ${ }^{13}$

Comme le dit très bien Edmond Jouve «la fonction gouvernementale serait alors indifférente à la nature de l'organe qui l'exerce». ${ }^{14}$ Il y a ici une différence d'extension de sens d'une langue à l'autre, la conception juridique française n'incluant jamais le législatif et le judiciaire dans la notion du gouvernement malgré le fait, comme nous l'avons dit ci-dessus, qu'on reconnaisse à ce dernier certaines compétences administratives.

À propos du type d'organes envisagés, si on considère uniquement le pouvoir exécutif, la notion anglaise recouvre non seulement l'ensemble des organes directeurs de ce pouvoir, mais aussi les organismes administratifs d'exécution des lois, et notamment les

12. Webster's Third New International Dictionary of the English Language - Unabridged (Springfield, Mass., G. and C. Merriam Company, 1981), p. 9.52.

13. Edmond Jouve, op. cit., p. 722. V. aussi: R. Mac Gregor Dawson and W.F. Dawson, Democratic Government in Canada, Fourth Edition (Toronto, University of Toronto Press, 1971), pp. 7-8.

14. Edmond Jouve, ibid. 
ministères ${ }^{15}, c^{\prime}$ 'est-à-dire ce qu'on désigne en langage juridique français sous le nom d'organes administratifs. Comme nous l'avons fait remarquer ci-dessus, ce n'est que dans le langage courant que la langue française établit cette adéquation.

À la distinction entre les champs notionnels des termes «gouvernement» et «government» se superpose une similitude de sens lorsqu'on introduit dans l'analyse notionnelle un facteur socio-culturel qui est celui du niveau de langue. Cette constatation ne manquera pas d'avoir des conséquences importantes pour le traducteur qui doit rendre ces notions par le terme adéquat.

\section{La polysémie du terme «administration» en anglais}

Ce qui rend le problème de l'équivalence notionnelle encore plus complexe, c'est l'extrême polysémie du terme «administration» en anglais.

A. Dunsire recense quinze sens différents de ce terme après avoir dépouillé une documentation volumineuse. ${ }^{16}$ Nous n'en retiendrons que trois dans la mesure où notre propos se limite à l'établissement des rapports notionnels que ce terme entretient avec celui de «government».

Le terme «administration» désigne l'ensemble des acritivés d'un État dans l'exercice du pouvoir politique, incluant l'action de ses organes législatif, exécutif et judiciaire. En outre, il signifie la gestion des affaires publiques par opposition à la fonction exécutive d'élaboration des politiques. ${ }^{17}$

15. R. Mac Gregor Dawson, op. cit., chap. 9, pp. 75 et suiv. V. aussi: J.R. Mallory, The Structure of Canadian Governement, rev. ed, (Toronto, Gage Publishing Ltd., 1984), chap. 4, p. 126 et suiv.

16. A. Dunsire, Administration, the Word and the Science (Bristol, Western Printing Services Ltd., 1973).

17. Webster's Third New International Dictionary of the English Language - Unabridged, op. cit., p. 28. 
Dans le premier cas, il est synonyme de «government» et correspond à peu près à ce que nous avons analysé en français sous le terme de «fonction gouvernementale» mais avec l'extension de sens propre à l'usage américain incluant les trois pouvoirs. Dans le deuxième cas, comme en français, il correspond à la fonction administrative d'application des politiques générales émanant des instances suprêmes du pouvoir exécutif. Le premier sens est courant aux États-Unis (the Bush Administration) tandis que la tradition britannique, on utilise le terme «government» (the Mulroney Government).

Outre ces deux sens fonctionnels, «administration» possède aussi un sens organique recouvrant l'ensemble des ministères et des bureaux de l'administration publique. Pour reprendre les termes d'A. Dunsire: «a collective noun for the non-judicial machinery of civil offices $\gg .{ }^{18}$ Il est intéressant de noter que dans l'ouvrage de cet auteur, d'origine britannique, les tribunaux ne sont pas considérés comme des organes du gouvernement. Comme nous venons de le voir, la situation est différente aux États-Unis en raison d'une conception différente de la séparation des pouvoirs. Selon cette conception, en régime présidentiel, les trois pouvoirs sont nettement séparés mais ils sont tous regroupés sous la notion de «government», tandis qu'en régime parlementaire européen, l'exécutif et le législatif, bien que séparés, entretiennent des liens plus étroits l'un avec l'autre. Ils sont regroupés dans une catégorie à part par rapport au judiciaire.

Cet exemple nous permet de constater que l'extension de ces notions varie non seulement selon les niveaux de langue mais aussi d'après les régimes et les traditions juridiques en vigueur là où ces notions sont utilisées.

Les rapports entre les notions de «government» et d'«administration» en anglais

Comme nous venons de le voir, «government» et «administration» apparaissent, dans certains cas, comme de véritables synonymes. Non

18. A. Dunsire, op. cit., p. 228. 
seulement «administration» est synonyme de «government» lorsqu'il désigne l'ensemble des activités d'un État dans l'exercice du pouvoir politique mais, en langage courant, on utilise aussi le terme «government» pour exprimer le sens organique du terme «administration» lorsqu'on se réfêre à l'ensemble des ministères et des bureaux de l'administration. De plus, on utilise «administration», aux États-Unis, pour désigner le groupe de personnes exerçant le pouvoir exécutif qu'on désigne en Angleterre et au Canada sous le vocable «government». A. Dunsire relève un autre usage britannique du terme «administration»: «a collective noun for the King's ministers, without the King; the government of the day», ${ }^{19}$ notion à laquelle on réserve habituellement le terme «government».

Il faut ajouter à cela que la fonction gouvernementale et la fonction administrative sont liées entre elles dans l'exercice quotidien de leurs prérogatives et au niveau des personnes qui les représentent au point que, au-delà des distinctions terminologiques qui les caractérisent dans le vocabulaire juridique, une certaine confusion linguistique s'est emparée de l'utilisateur qui désire exprimer ces notions. Comme le dit très bien Herbert Kaufman: «the functions of administration may be considered virtually as broad as the functions of government». ${ }^{20}$

\section{Conclusion}

L'extrême malléabilité de ces termes, dans chacune des deux langues, nous oblige à les examiner dans leur contexte particulier pour en déterminer le sens et en effectuer la traduction la plus appropriée lorsqu'ils sont mis en présence les uns des autres. Il n'est pas étonnant que les traducteurs juristes du gouvernement fédéral canadien ont traduit «governmental function» par «fonction administrative» dans la loi dont nous venons de faire état ci-dessus. Le contexte étant manifestement d'ordre juridique, nous nous devons d'écarter toute désignation provenant de la langue courante pour rendre le terme anglais. De plus,

19. A. Dunsire, ibid.

20. International Encyclopedia of the Social Sciences, David L. Sills, ed., vol. 1 (New York, Mac Millan, 1968), p. 63. 
il apparaît clairement que cette fonction est celle qui est remplie par un ensemble d'organismes administratifs chargés de l'application des décisions gouvernementales de l'État. Ces organismes, au sens propre du terme, ne peuvent qu'exercer une fonction administrative selon la distinction traditionnelle que nous avons exposée ci-dessus et non une fonction de politique générale. C'est, par conséquent, le terme «fonction administrative», au sens juridique, qui doit être utilisé et nous avons intérêt à écarter les emplois particuliers du terme "gouvernement» en français.

Lorsqu'il a affaire à des vocables de ce genre, le traducteur devra non seulement tenir compte du contexte immédiat que lui fournit le document à traduire, mais encore du niveau de langue de l'utilisateur du texte et de son auteur. Il devra, de plus, être particulièrement attentif au contexte juridique dans lequel il s'inscrit. Nous pouvons d'autre part affirmer, en nous fondant sur une longue expérience de la recherche en terminologie de la gestion et de l'administration publique, que dans ces domaines, ce sont les termes apparemment les plus simples qui posent le plus de problèmes au traducteur.

Cette étude nous permet aussi d'apprécier l'utilité de la terminologie comparée dans la résolution des difficultés de traduction liées à la présence de faux amis dans deux langues ayant une origine commune. Nous sommes, en effet, en présence de deux paires de termes qui ont la même forme dans chaque langue mais dont les sens diffèrent.

L'établissement d'une équivalence correcte dépend de l'analyse du champ notionnel de chaque groupe de notions, dans chacune des deux langues, selon les méthodes de la terminologie unilingue. Ensuite, les recoupements notionnels potentiels sont envisagés dans une perspective comparatiste. Il appartient alors au traducteur de faire le choix qui s'impose.

École de traducteurs et d'interprètes Université d'Ottawa 